\section{The Impact of Standardizing Assessment and Treatment of Acute Asthma Exacerbations on Emergency Department Efficiency}

\author{
John S Garrett ${ }^{1 *}$, Yahya A Daoud ${ }^{2}$, Eugene Gicheru1, James \\ D'Etienne ${ }^{1}$, Mary K Hart ${ }^{3}$ and Vickey Thompson ${ }^{1}$
}

${ }^{1}$ Department of Emergency Medicine, Baylor University Medical Center, Dallas, Texas, USA

${ }^{2}$ Department of Quantitative Sciences, Institute for Health Care Research and Improvement, Baylor Healthcare System, Dallas, Texas, USA

${ }^{3}$ Department of Respiratory Care, University of Texas Health Science Center of San Antonio, Texas, USA

\begin{abstract}
Background: Emergency Department (ED) based Acute Asthma Exacerbation (AAE) clinical pathways effectively improve patient assessment, drug therapy, patient education, and discharge instructions. The effect of such protocols on hospital admission rates and ED length of stay are unclear.

Objectives: Evaluate the impact of a standardized Asthma Treatment Protocol (ATP) on ED efficiency and return to the ED within 24 hours.

Methods: This was a retrospective cohort analysis of 240 randomly selected patients presenting to the ED with AAE during the 3 months preceding and following the institution of an ATP. The primary outcome was the average Length of Stay (LOS) between Pre-ATP and ATP cohorts in patients discharged from the ED.

Results: During the study 763 patients left the ED with a diagnosis of AAE. One hundred twenty cases were randomly selected from each cohort and reviewed to exclude secondary causes of dyspnea. There was no significant difference in AAE severity between the two groups. Despite this, the ATP cohort was more likely to be admitted to the hospital than the pre-ATP cohort $(31.8 \%$ vs $11.7 \%, p<0.004)$. Though no difference was noted in the LOS in patients discharged home from the ED between the ATP and pre-ATP cohorts (223 minutes $S D=113$ versus 219 minutes $S D=105, p=0.840$ ), the significant increase in admission rate led to an overall increase in
\end{abstract}

*Corresponding author: John S Garrett, Department of Emergency Medicine Baylor University Medical Center, Dallas, Texas, USA, Tel: +1 2145990594 ; E-mail: John.Garrett@baylorhealth.edu

Citation: Garrett JS, Daoud YA, Gicheru E, D’Etienne J, Hart MK, et al. (2016) The Impact of Standardizing Assessment and Treatment of Acute Asthma Exacerbations on Emergency Department Efficiency. J Emerg Med Trauma Surg Care 3: 013.

Received: December 04, 2015; Accepted: January 07, 2016; Published: January 21, 2016
OS that was not statically significant (273 minutes $(S D=134)$ versus 244 minutes $(S D=141, p=0.21)$. More patients received disease specific asthma education in the ATP group than the pre-ATP group ( $79.1 \%$ vs $11.7 \%, p<0.0001)$.

Conclusion: Institution of a standardized asthma treatment protocol appears to increase Emergency Department efficiency and allow for patient education and discharge planning in patient discharged from the ED, however it may increase the percentage of patients admitted to the hospital.

Keywords: Asthma; Efficiency; Emergency Department; Length of Stay; Protocol

\section{Introduction}

Despite advances in outpatient management, Acute Asthma Exacerbation (AAE) remains a common cause for presentation to the Emergency Department (ED), accounting for approximately 1.8 million visits each year [1]. $10-20 \%$ of these visits require admission to the hospital for further treatment and management [2,3]. Multiple treatment modalities have been demonstrated to significantly decrease the severity of AAE and reduce the need for hospital admission [4]. The National Institute of Health (NIH) guidelines published in 2007 suggest a multidisciplinary approach to optimize treatment of AAE within the ED [4]. These include suggestions on clinical assessment, objective measurement of episode severity, appropriate and timely treatment while in the ED. It also calls for appropriate follow-up arrangements, asthma education, and a written asthma action plan for patients amenable to discharge.

In the hospital, multidisciplinary clinical pathways which include nursing and respiratory care have been effective in reducing hospital length-of-stay and inpatient costs [5]. The effect of similar protocols for the treatment of AAE within the ED have been described, and have been objectively evaluated within the adult population [6-8]. Several studies have shown ED asthma guidelines have been effective in improving appropriate patient assessment, drug therapy, and patient education [9-11]. However, the effect of such protocols on rates of admission to the hospital and ED Length of Stay (LOS) are unclear. Clinical pathways would be expected to improve efficiency, utilization of resources, and quality of clinical care in a busy, high-volume ED. This is balanced by the time required to provide patient asthma education and follow-up.

Based upon NIH guidelines we instituted a multidisciplinary asthma protocol (Figure 1). This new protocol streamlined clinical care and instituted four new interventions: initiation of nebulized short acting agonists (SABA) prior to or concurrent with physician evaluation, objective pre and post treatment measurements of AAE severity though the use of percent predicted Forced Expired Volume 1 (ppFEV1), SABA administration via breath actuated nebulizer rather than traditional continuous flow nebulizers [12], and the development and implementation of an individual Asthma Action Plan (Figure 2) for discharged patients. It was hypothesized implementation of a standardized Asthma Treatment Protocol (ATP) within the ED will improve average ED LOS and prevent return visits to the ED within 24 hours. 


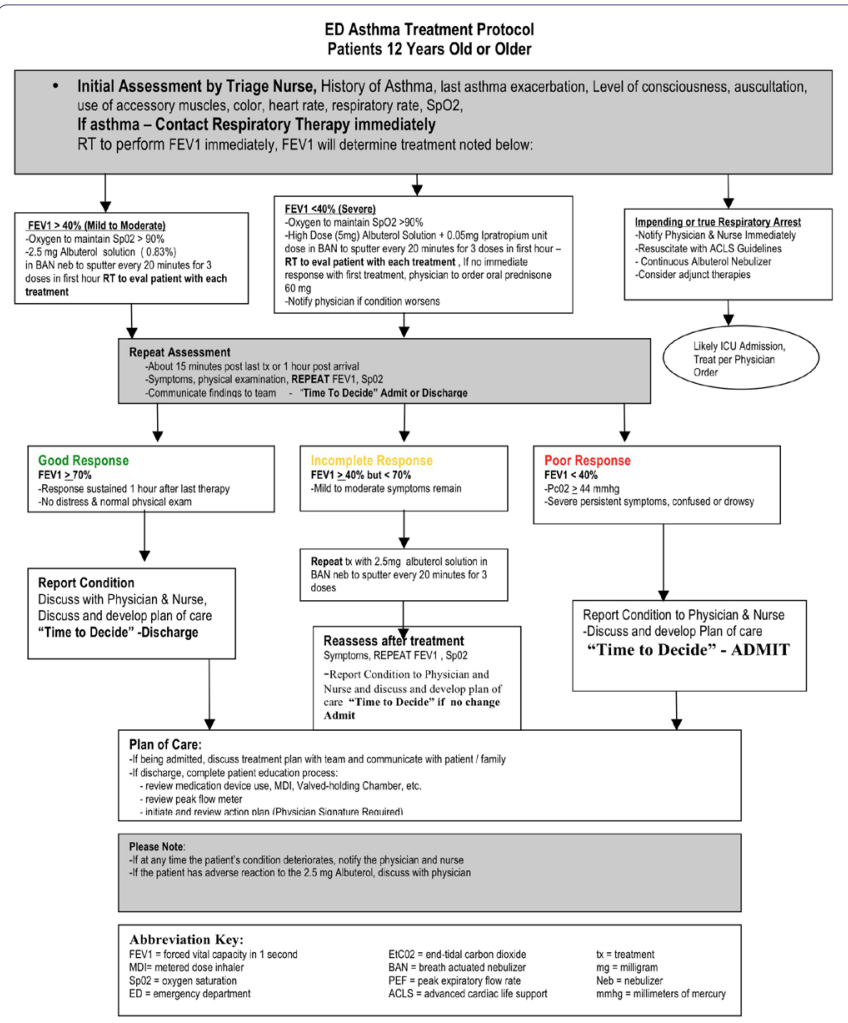

Figure 1: ED Asthma treatment protocol patients 12 year old or older.

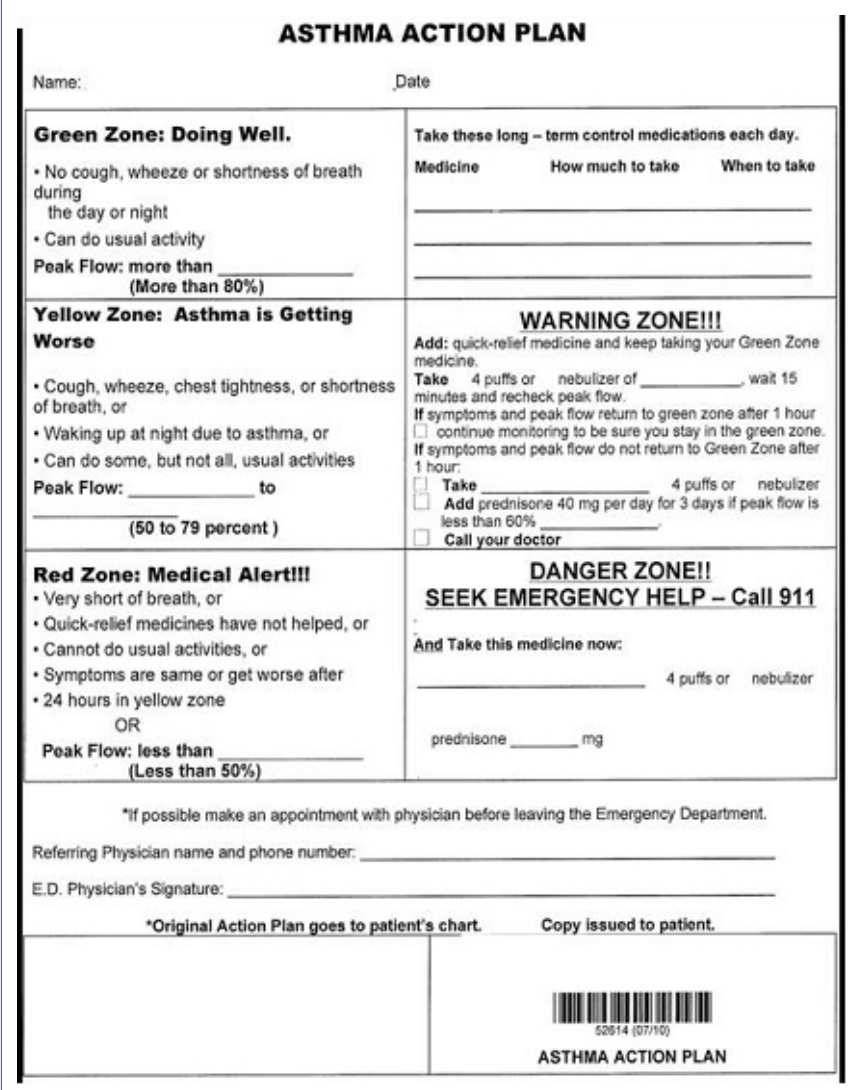

Figure 2: Asthma action plan.

\section{Materials and Methods}

This was a retrospective, single center, pre/post cohort analysis of patients discharged or admitted from the ED with a diagnosis acute exacerbation of asthma. On 12/1/2008 the ATP was instituted at our institution, a large (>100,000 visits/year) tertiary care center. The multidisciplinary protocol involved changes to nursing, respiratory, and physician practice patterns. As such, a 6 month training period was allowed to teach and become acclimated to the new protocol. All patients age 12 years and greater who presented to the ED and diagnosed with an AAE during the 3 months preceding the institution of the protocol (pre-ATP) and during the 3 months following the training period were eligible for inclusion in the study. Patients with secondary etiologies of dyspnea (pneumonia, pulmonary edema, pulmonary embolism, acute coronary syndrome, and significant anemia) were excluded. 120 patients from each cohort were randomly selected via random number generator for chart review to determine secondary etiologies of dyspnea and coexisting medical conditions. An acute asthma exacerbation was defined as a progressively worsening of dyspnea, cough, wheezing, or chest tightness secondary to bronchospasm as determined by the attending Emergency Medicine physician at the time of treatment.

Demographic and clinical features were collected in all eligible patients to compare severity of initial illness between groups. Additionally, treatment specifics such as the dose of nebulized SABA (Albuterol/Xopenex) administered, steroid dose, magnesium use, as well as the initiation of respiratory support (BiPAP and endotracheal intubation) were recorded. The use of Asthma Education and Asthma Action plan at the time of discharge was recorded to ensure compliance with the more education intensive aspects of the protocol. Evaluation of the completion of asthma education was noted based upon documentation of individual instruction on use of a peak flow meter, aerochamber, asthma diary, or medications by the respiratory technician, nurse, or physician. LOS was defined as the difference in time between arrival at the ED and disposition from the ED (home or to the inpatient unit/ICU).

The primary outcome was the decrease in average LOS between Pre-ATP and Post-ATP cohorts in patients discharged from the ED. Secondary outcomes include decrease in the rate of return to the ED within 24 hours and the time interval between arrival and treatment with SABA. The primary independent variable of interest was the ATP. The dichotomous variable was patients classified as either receiving the standard asthma care prior to instituting the ATP or receiving the instituted ATP

\section{Data analysis}

All data analyses were performed using JMP' 10 (SAS Institute Inc., Cary, NC, USA) on an intention to treat basis. Presentation characteristics and demographic data were analyzed using frequencies, and percentages for categorical variables such as gender, race method of arrival, means, and standard deviations for continuous variables. Where appropriate, tests for differences in population characteristics were performed using chi-squared analysis or t-tests. Primary data analysis focused on a two group comparison of use of ATP compared to the continuous variable LOS and compared with student $\mathrm{t}$-tests. Rates of admission to the hospital and return the ED within 24 hours were evaluated using $\mathrm{c}^{2}$ tests. Multivariate regression was utilized to evaluate the relationship between LOS and the covariates. 


\begin{tabular}{|c|c|c|c|}
\hline & \multicolumn{2}{|c|}{ Status } & \multirow[b]{2}{*}{ P-Value } \\
\hline & $\begin{array}{c}\text { Pre-ATP } \\
(\mathrm{n}=77)\end{array}$ & $\begin{array}{c}\text { ATP } \\
(n=66)\end{array}$ & \\
\hline Gender & $45(58.4 \%)$ & $23(34.8 \%)$ & 0.007 \\
\hline $\begin{array}{r}\text { Black } \\
\text { White } \\
\text { Others }\end{array}$ & $\begin{array}{c}55(71.4 \%) \\
15(19.5 \%) \\
7(9.1 \%)\end{array}$ & $\begin{array}{c}50(75.8 \%) \\
15(22.7 \%) \\
1(1.5 \%)\end{array}$ & 0.141 \\
\hline $\begin{array}{r}\text { EMS } \\
\text { Walk-in }\end{array}$ & $\begin{array}{l}17(22.1 \%) \\
59(76.6 \%)\end{array}$ & $\begin{array}{l}9(13.6 \%) \\
55(83 \%)\end{array}$ & 0.276 \\
\hline Pre-hospital Treatment & $19(24.7 \%)$ & $7(10.6 \%)$ & 0.009 \\
\hline Was there an order for "Asthma Protocol" & $0(0.0 \%)$ & $46(69.7 \%)$ & $<0.0001$ \\
\hline $\begin{array}{r}\text { \# of SABA Treatments } \\
\text { One } \\
\text { Two } \\
\text { Three or More }\end{array}$ & $\begin{array}{l}18(23.4 \%) \\
24(31.2 \%) \\
35(45.2 \%)\end{array}$ & $\begin{array}{l}33(64.7 \%) \\
20(45.5 \%) \\
13(27.1 \%)\end{array}$ & 0.001 \\
\hline Respiratory Support? (BiPAP, ETI) & $1(1.3 \%)$ & $1(1.5 \%)$ & 1.000 \\
\hline $\begin{array}{l}\text { Baseline HR } \\
\qquad \text { Average beats/minute (SD) }\end{array}$ & $96.3(22.23)$ & $105(24.83)$ & 0.028 \\
\hline $\begin{array}{l}\text { Baseline SBP } \\
\qquad \text { Average } \mathrm{mmHg}(\mathrm{SD}) \\
\end{array}$ & $134.6(24.2)$ & $143.1(27.4)$ & 0.067 \\
\hline $\begin{array}{l}\text { Baseline } 02 \text { Sat } \\
\text { Average \% Saturation (SD) }\end{array}$ & $96.8(2.8)$ & $97.0(2.7)$ & 0.654 \\
\hline $\begin{array}{l}\text { DC HR } \\
\quad \text { Average beats/minute (SD) }\end{array}$ & $96.3(21.7)$ & $105.9(20.8)$ & 0.011 \\
\hline DC SBP & $126.0(18.7)$ & $128.0(17.1)$ & 0.540 \\
\hline Average \% Saturation (SD) & $97.6(2.2)$ & $98.2(1.8)$ & 0.092 \\
\hline Time to treat & $68.5(69.33)$ & $56.8(54.2)$ & 0.260 \\
\hline $\begin{array}{l}\text { Pre FEV } 1 \text { (\% predicted or liters) } \\
\quad \text { Average (SD) }\end{array}$ & $53.5(22.8)$ & $45.8(22.7)$ & 0.150 \\
\hline
\end{tabular}

This study was approved by the Institutional Review Board of the Baylor Health Care System. The authors do not report any conflicts of interest.

\section{Results}

During the study period 763 patients were discharged or admitted with a final diagnosis of acute asthma exacerbation. Overall ED census increased by $6.2 \%$ (4714 to 5006 ) during the study period. AAE census increased $23.9 \%$ (343 patients to 425 patients) in the 3 months preceding institution of the ATP versus the 3 months after the ATP training period. From this larger cohort, 120 patients were randomly selected from each group for chart review and final data analysis. After excluding the 97 patients who had secondary causes of dyspnea, 66 (46.2\%) remained in the ATP group and 77 (53.8\%) remained in the
pre-ATP group. Of the patients within the ATP group $60 \%$ of patients completed the entire ATP with serial ppFEV1, asthma education and action plan, $30 \%$ received part of the protocol with a single ppFEV1 recorded, and $10 \%$ did have a ppFEV1 measured.

Demographic and characteristics of the initial clinical presentation of the study population are shown in table 1. There was no statistically significant difference between the two groups of patients related to asthma exacerbation severity. Patients in the pre-ATP group were more likely to be male, have received pre-hospital SABA treatment, and had a lower initial heart rate. Overall LOS in patient who received care dictated by the ATP was 273 minutes $(\mathrm{SD}=134)$ versus 244 minutes $(\mathrm{SD}=141, \mathrm{p}=0.21)$ in those who did not. Admission rate increased from $11.7 \%$ to $31.8 \%(\mathrm{p}<0.004)$ from the pre-ATP group to the ATP group. LOS in those patients discharged 


\begin{tabular}{|c|c|c|c|}
\hline \multirow[b]{2}{*}{ Measurements } & \multicolumn{2}{|c|}{ Status } & \multirow[b]{2}{*}{ P-Value } \\
\hline & $\begin{array}{l}\text { Pre-ATP } \\
(\mathrm{n}=77)\end{array}$ & $\begin{array}{c}\text { ATP } \\
(n=66)\end{array}$ & \\
\hline \multicolumn{4}{|l|}{ Average Length of Stay (SD) } \\
\hline Total & $244.3(140.6)$ & $273.0(134.3)$ & 0.210 \\
\hline Admit to the Hospital & $437.33(219.2)$ & $379.9(113.6)$ & 0.470 \\
\hline Discharge from the ED & $218.78(105.0)$ & $223.2(113.2)$ & 0.840 \\
\hline \multicolumn{4}{|l|}{ Return to the ED within 24 hours (\%) } \\
\hline Yes & $1(1.3 \%)$ & $0(0.0 \%)$ & 1.0000 \\
\hline \multicolumn{4}{|l|}{$\begin{array}{l}\text { Documentation of Asthma Education (peak flow, med delivery } \\
\text { device, aerochamber, patient diary) }\end{array}$} \\
\hline Yes & $9(11.7 \%)$ & $34(79.1 \%)$ & $<0.0001$ \\
\hline Yes & $9(11.7 \%)$ & $21(31.8 \%)$ & 0.004 \\
\hline
\end{tabular}

from the ED who received care as dictated by the ATP was 223 minutes $(\mathrm{SD}=113)$ versus 219 minutes $(\mathrm{SD}=105.0, \mathrm{p}=0.840)$ in those who did not. No difference in LOS was seen in the subgroups of those patients admitted or discharged from the ED (Table 2) though there was a non-statistically significant trend towards increase overall LOS in the ATP group related to an increase in admission rate.

Multivariate regression showed LOS was significantly associated with the need for admission to the hospital, time from triage to first treatment, need for respiratory support, and number of treatments administered. Abnormal discharge heart rate and oxygen saturations were also associated with prolonged LOS.

\section{Discussion}

This retrospective study demonstrates that the provision of standardized multidisciplinary care for the treatment of acute asthma exacerbation does not increase LOS in a high volume tertiary care ED. Efficiency, quality of care, and patient satisfaction are important components of the overall medical care provided within the ED. In a busy ED setting it is often difficult to devote sufficient resources to allow for adequate patient education, discharge instructions, and follow-up. Several studies have evaluated patient comprehension of discharge instructions and found only $22 \%$ of patients discharged from the ED fully understand their discharge instructions $[13,14]$. This has negative ramifications on long-term care, patient satisfaction, and likely increases downstream use of the health care system $[15,16]$. Standardized intensive disease specific education likely helps to ameliorate the negative effects this may impose upon ED efficiency. In this study, the ATP was able to bundle early assessment, evaluation, treatment, and discharge education with instructions into an approach that did not impact LOS in discharged ED patients. Similar finding were described by Doherty et al., when implementing asthma guidelines in the Emergency Department [8].

Interestingly we saw an increase in the rate of admissions between the two groups as the ATP was utilized more frequently. A similar phenomenon was noted in the Lougheed et al., when they implemented an asthma care pathway across 5 hospital ED's. In their study, admission rates increased from 3.9 to $9.4 \%$ which constitutes an almost 2.5 fold increase. We saw a significant and similar 2.7 fold in the rate of admission (11.7 to 31.8) despite both patient populations having essentially the same initial attack severity as measured by pre-treatment ppFEV1. This may be due to more rapid identification and decision as to which patients require admission. The use of spirometry/FEV1 in the evaluation of acute asthma exacerbation has been shown to provide a more accurate determination of attack severity and clinical exam alone [17]. Additionally, repeated measurements of peak flow or ppFEV1 in adults at 1 hour and beyond are useful as isolated assessments in determining who will require hospitalization and who is likely to have sufficient response to treatment to allow continued ED care [18-21]. Indeed, repeated ppFEV1 or peak flow measures at presentation to the $\mathrm{ED}$ and 1 hour after treatment were the strongest single predictor of hospitalization among adults who present to the ED with an asthma exacerbation [22]. It is possible that as ATPs based upon ppFEV1 or peak flow increase the frequency of hospital admissions by designating patients as requiring admission whom would not have required admission based upon physical exam based assessments.

\section{Limitations}

This was a retrospective study and was inherently subject to a number of biases which may have influenced our results. One potential factor biasing our results could be due to the addition of the protocol itself. By adding increased awareness on early asthma treatment and recognition, it is possible our increase in efficiency was due to provider focus on the quality of care in the asthmatic patients. Similar to an observer bias, this may have resulted in more rapid assessment and treatment with the resultant improvement in our outcomes.

We were only able to track return visits to our system of hospitals. As such, it is possible the patients sought care at another institution after ED discharge. In this way our return visit rate may be under estimated, though the rate of this occurrence would likely be similar between the two groups. In a similar manner, per institution protocol 24 hour returns were tracked and considered treatment failures. Other studies have used longer windows to define outpatient treatment failure [23].

Additionally, it may be possible that there were differences in disease severity between our two groups. Each group presented at different time of year and as such it is possible that the etiology of the underlying trigger for the AAE was seasonal. To this effect, we saw an increase in the total number of patients who presented to the ED reporting AAE during the study period. However, objective measures of attack severity (vital signs, pretreatment ppFEV1, and need for respiratory support) were similar between the two groups. As such, 
it is unlikely this seasonal variation impacted admission rates given similar attack severity.

Finally, we evaluated the patients on an intention to treat basis. It is possible patients within the ATP cohort did not receive the full protocol. This would have had the overall effect of diminishing any appreciable gains in efficiency made by the ATP itself. Most patients in the ATP group (60\%) received the full protocol without omission, while an additional $30 \%$ received part of the protocol. A subsequent subgroup "as treated" analysis demonstrated a trend towards improved LOS in the treatment group when compared to the patients who did not received care dictated by the ATP though this was not statistically significant. This further suggests the ATP improved ED efficiency, though further study would be required to verify this finding.

\section{Conclusion}

Institution of a standardized asthma treatment protocol appears to increase Emergency Department efficiency and allow for patient education and discharge planning in patient discharged from the ED, however it may increase the percentage of patients admitted to the hospital.

\section{References}

1. Rowe BH, Voaklander DC, Wang D, Senthilselvan A, Klassen TP, et al (2009) Asthma presentations by adults to emergency departments in Alberta, Canada: a large population based study. Chest 135: 57-65

2. Pollack CV Jr, Pollack ES, Baren JM, Smith SR, Woodruff PG, et al. (2002) A prospective multicenter study of patient factors associated with hospital admission from the emergency department among children with acute asthma. Arch Pediatr Adolesc Med 156: 934-940.

3. Weber EJ, Silverman RA, Callaham ML, Pollack CV, Woodruff PG, et al (2002) A prospective multicenter study of factors associated with hospital admission among adults with acute asthma. Am J Med 113: 371-378.

4. US Department of Health and Human Services (2007) National Asthma Education and Prevention Program. Expert Panel Report 3: Guidelines for the diagnosis and management of asthma. US Department of Health and Human services, National Heart, Lung, and Blood Institute, National Institutes of Health.

5. Banasiak NC, Meadows-Oliver M (2004) Inpatient asthma clinical pathways for the pediatric patient: an integrative review of the literature. Pediatr Nurs 30: $447-450$.

6. Kanis J, Lovell J, Bowman M, Titus MO (2011) Focused assessment of patients with asthma in the emergency department. Clin Pediatr (Phila) 50: 529534.

7. Lougheed MD, Olajos-Clow J, Szpiro K, Moyse P, Julien B, et al. (2009) Multicentre evaluation of an emergency department asthma care pathway for adults. CJEM 11: 215-229.
8. Doherty SR, Jones PD, Davis L, Ryan NJ, Treeve V (2007) Evidence-based implementation of adult asthma guidelines in the emergency department: $A$ controlled trial. Emerg Med Australas 19: 31-38.

9. Chouaid C, Bal JP, Fuhrman C, Housset B, Caudron J (2004) Standardized protocol improves asthma management in emergency department. J Asthma 41: $19-25$.

10. Cabana MD, Bruckman D, Bratton SL, Kemper AR, Clark NM (2003) Association between outpatient follow-up and pediatric emergency department asthma visits. J Asthma 40: 741-749.

11. Lougheed MD, Olajos-Clow J, Szpiro K, Moyse P, Julien B, et al. (2009) Multicentre evaluation of an emergency department asthma care pathway for adults. CJEM 11: 215-229.

12. Sabato K, Ward P, Hawk W, Gildengorin V, Asselin JM (2011) Randomized controlled trial of a breath-actuated nebulizer in pediatric asthma patients in the emergency department. Respir Care 56: 761-770.

13. Zavala S, Shaffer C (2011) Do patients understand discharge instructions? J Emerg Nurs 37: 138-140.

14. Engel KG, Heisler M, Smith DM, Robinson CH, Forman JH, et al. (2009) Patient comprehension of emergency department care and instructions: are patients aware of when they do not understand? Ann of Emerg Med 53: 454461.

15. Chugh A, Williams MV, Grigsby J, Coleman EA (2009) Better transitions: improving comprehension of discharge instructions. Front Health Serv Manage 25: $11-32$.

16. McCarthy DM, Engel KG, Buckley BA, Forth VE, Schmidt MJ, et al. (2012) Emergency Department Discharge Instructions: Lessons Learned through Developing New Patient Educations Materials. Emerg Med Int 2012: 1-7.

17. Karras DJ, Sammon ME, Terregino CA, Lopez BL, Griswold SK, et al. (2000) Clinically meaningful changes in quantitative measures of asthma severity. Acad Emerg Med 7: 327-334.

18. Kelly AM, Kerr D, Powell C (2004) Is severity assessment after one hour of treatment better for predicting the need for admission in acute asthma? Respir Med 98: 777-781.

19. McCarren M, Zalenski RJ, McDermott M, Kaur K (2000) Predicting recovery from acute asthma in an emergency diagnostic and treatment unit. Acad Emerg Med 7: 28-35.

20. Rodrigo G, Rodrigo C (1993) Assessment of the patient with acute asthma in the emergency department. A factor analytic study. Chest 104: 1325-1328.

21. Rodrigo G, Rodrigo C (1998) Early prediction of poor response in acute asthma patients in the emergency department. Chest 114: 1016-1021.

22. Weber EJ, Silverman RA, Callaham ML, Pollack CV, Woodruff PG, et al. (2002) A prospective multicenter study of factors associated with hospital admission among adults with acute asthma. Am J Med 113: 371-378.

23. Emerman CL, Woodruff PG, Cydulka RK, Gibbs MA, Pollack CV Jr, et al. (1999) Prospective multicenter study of relapse following treatment for acute asthma among adults presenting to the emergency department. MARC investigators. Multicenter Asthma Research Collaboration. Chest 115: 919-927. 